\title{
Simultaneous XMM-Newton and BeppoSAX observation of the Seyfert I galaxy IC 4329A
}

\author{
P. Gondoin, P. Barr, D. Lumb, T. Oosterbroek, A. Orr, and A. N. Parmar \\ Astrophysics Division, European Space Agency - Postbus 299, 2200 AG Noordwijk, The Netherlands
}

Received 1 June 2001 / Accepted 6 September 2001

\begin{abstract}
We report on a simultaneous XMM-Newton and BeppoSAX observation of the Seyfert 1 galaxy IC 4329A performed in 2001 January. Above $1 \mathrm{keV}$, EPIC spectra show a narrow Fe K emission line at $6.42_{-0.03}^{+0.04} \mathrm{keV}$ with an equivalent width of $43 \pm 1 \mathrm{eV}$. An absorption edge is detected at $7.1 \pm 0.4 \mathrm{keV}$ in the source rest frame that has not been reported before. The energy of these features is consistent with reflection of the primary X-ray continuum by a optically thick material containing iron in low states of ionization. The narrow Fe $\mathrm{K}$ feature suggests that the reflection arises relatively far from a putative central black hole. Spectral fitting of simultaneous XMM-Newton and BeppoSAX data over the $0.3-130 \mathrm{keV}$ energy range shows that the primary continuum is well described by a cut-off power law with a photon index of $1.93 \pm 0.03$ and a folding energy of $260 \pm 130 \mathrm{keV}$. The high energy tail $>10 \mathrm{keV}$ constrains the reflection fraction close to unity. Fitting over the overall spectral range indicates that the Fe abundance within the cool reflecting material is not significantly different from the cosmic value. The extrapolation of the primary power law continuum to energies $<1 \mathrm{keV}$ indicates the presence of absorption edges produced by neutral and ionized oxygen. The optical depth of the $\mathrm{O} I$ edge at $0.54 \mathrm{keV}$ constrains the intrinsic absorption by neutral material to an hydrogen column density of $=(33 \pm 3) \times 10^{20} \mathrm{~cm}^{-2}$ along the line of sight to IC $4329 \mathrm{~A}$ nucleus. The energy of the edge at $0.854 \pm 0.009 \mathrm{keV}$ is consistent with absorption by O VIII. A third edge at $0.660 \pm 0.008 \mathrm{keV}$ is likely related to absorption by O VI and N VII. The simultaneous presence of O VI and O VIII edges without significant O VII absorption indicates that the warm absorber originates from at least two zones.
\end{abstract}

Key words. galaxies: individual: IC 4329A - galaxies: nuclei - galaxies: Seyfert

\section{Introduction}

IC $4329 \mathrm{~A}$ is a nearby Seyfert 1 galaxy (Whittle 1992), at a redshift $z=0.016$ (Wilson \& Penston 1979). X-ray observations show a power law spectrum with a reflection component which flattens the photon index of the primary component in the 1.7 to $30 \mathrm{keV}$ energy band (Miyoshi et al. 1988; Piro et al. 1990). The reflection component and the iron K $\alpha$ line present in IC 4329A spectra (Piro et al. 1990; Perola et al. 1999) are likely the result of X-ray reprocessing mechanisms by material within the inner region of an accretion disk. By analysing these spectral components in high signal to noise data covering a wide spectral range from $0.3 \mathrm{keV}$ to $130 \mathrm{keV}$, the present study aims at new information about the geometry and the nature of the physical processes which operate in the immediate environment of the IC 4329A central engine. The iron line, in particular, is an important tool for mapping the inner regions of the putative accretion disk. Using ASCA observations of IC 4329A performed in 1997 August,

Send offprint requests to: $\mathrm{P}$. Gondoin,

e-mail: pgondoin@astro.estec.esa.nl
Done et al. (2000) found that the Fe K line of IC 4329A is significantly broadened, but not to the extent expected from an accretion disk that extends down to the last stable orbit around a black hole. Such evidence is not found in all AGNs. In many objects, lines are narrower than $1000 \mathrm{~km} \mathrm{~s}^{-1}$ across and vary in flux far more slowly than the continuum, suggesting that they originate far from a putative central black hole (Weaver et al. 1996; Guainazzi et al. 1998; Yaqoob et al. 2001; Gondoin et al. 2001).

In the soft energy range, the X-ray spectra of many AGNs deviate from a simple power law continuum absorbed by Galactic material. The presence of a soft X-ray excess has been established in a large fraction of Seyfert galaxies (Turner \& Pounds 1989; Walter \& Fink 1994). A significant fraction of type 1 active galactic nuclei (Reynolds 1997) also show K-shells absorption edges of warm oxygen (O VII and O VIII) characteristic of optically thin, photoionized material along the line of sight to the central engine, the so-called warm absorber. In the soft energy spectrum of IC 4329A, Madejski et al. (1995) discovered with ROSAT the existence either of a ionized edge imprinted by a warm absorber, or of a soft excess. 
The ambiguity was resolved by Cappi et al. (1996), who found in ASCA data evidence of two strong edges, consistent with O VII and O VIII. The ASCA data were further investigated by Reynolds (1997) and George et al. (1998). From a spectral analysis of a broadband BeppoSAX observation of IC 4329A, Perola et al. (1999) reported two absorption features at $0.7 \mathrm{keV}$ and $1 \mathrm{keV}$ corresponding to a blend of O VI and O VII and to a combinaton of Fe L and Ne K edges. The limitation in bandwidth and resolution of previous instruments and the presence of several components in the X-ray spectrum makes the origin of the soft excess and warm absorber still unclear. A good determination of the shape of the intrinsic power law, which should take into account the presence of a reflection component at high energy, is particularly important for measuring departure from the power law spectrum in the low energy band. In this respect, the present study of IC 4329A benefits from the high signal provided by the large collecting area of the $X M M-N e w t o n$ X-ray telescopes, from the good spectral resolution of the CCDs cameras and reflection grating spectrometers and from the wide spectral band coverage of the simultaneous BeppoSAX observations.

In this paper, we report the analysis results of X-ray observations of IC 4329A performed in January 2001. Section 2 details the observations and data reduction procedures. Section 3 presents the integrated flux measurements and their temporal behaviour during the observations. Section 4 describes the spectral analysis which was performed in two steps including first a characterization of the reflection components above $2 \mathrm{keV}$ and then an analysis of the soft $(0.3-2 \mathrm{keV})$ energy range. The analysis results are discussed in Sect. 5 .

\section{Observations and data reduction}

\subsection{XMM-Newton observations}

IC $4329 \mathrm{~A}$ was observed by the $X M M-N e w t o n$ space observatory (Jansen et al. 2001) on 2001 January 31 between 14:14:12 (UT) and 18:11:59 (UT) for usable exposure times of $10.9 \mathrm{ksec}$ (EPIC p-n camera), $13.0 \mathrm{ksec}$ (EPIC MOS 1 and MOS 2 cameras) and $13.9 \mathrm{ksec}$ (RGS 1 and RGS 2 Reflection Grating Spectrometers). XMMNewton uses three grazing incidence telescopes which provide an effective area $>4000 \mathrm{~cm}^{2}$ at $2 \mathrm{keV}$ and $1600 \mathrm{~cm}^{2}$ at $8 \mathrm{keV}$ (Gondoin et al. 2000). Three EPIC CCD cameras (Turner et al. 2001) at the prime focus of the telescopes provide imaging in a $30^{\prime}$ field of view and broadband spectroscopy with a resolving power of between 5 and 60 in the energy range 0.1 to $10 \mathrm{keV}$. Two identical RGS reflection grating spectrometers behind two of the X-ray telescopes allow high resolution $(E / \Delta E=100$ to 500) measurements in the soft X-ray range ( 6 to $38 \AA$ or 0.3 to $2.1 \mathrm{keV}$ ) with a maximum effective area of about $140 \mathrm{~cm}^{2}$ at $15 \AA$ (den Herder et al. 2001).

IC 4329A observations were conducted with the EPIC $\mathrm{p}-\mathrm{n}$ camera operating in full frame mode and with the EPIC MOS cameras operating in small window mode.
RGS spectra were recorded simultaneously. "Medium" thickness aluminum filters were used in front of all CCD cameras to reject visible light. Processing of the raw event data sets was performed using the "emproc", "epproc" and "rgsproc" pipeline tasks of the XMM-Newton Science Analysis System (SAS version 5.0.1). The large count rate of the target produced pile-up effects in the core of the telescope point spread functions registered by the EPIC cameras. In order to reject these ambiguous events, the source spectra in the EPIC cameras were built from photons detected within an annulus of radius included between $22^{\prime \prime}$ and $106^{\prime \prime}$ from the target boresight. The background was estimated on the same CCD chips within windows of similar size which were offset by about $2.5^{\prime}$ from the source centroid position. Background rates were found to be extremely low during the whole observation. The Pulse-Invariant (PI) spectra were rebinned such that each resultant channel had at least 100 counts per bin. $\chi^{2}$ minimization was used for spectral fitting. All such fits were performed using the XSPEC package (v11). EPIC response matrices provided by the PI institutes were used. The RGS response matrices were generated by the SAS task "rgsrmfgen".

\subsection{BeppoSAX observations}

IC 4329A was simultaneously observed between 2001 January 31 04:23 UT and February 02 02:131 UT by the BeppoSAX satellite (Boella et al. 1997). Results from the Low-Energy Concentrator Spectrometer (LECS; 0.1-10 keV; Parmar et al. 1997), the Medium-Energy Concentrator Spectrometer (MECS; 1.8-10 keV; Boella et al. 1997), and the Phoswich Detection System (PDS; 15-300 keV; Frontera et al. 1997) were analysed. The MECS consists of two grazing incidence telescopes with imaging gas scintillation proportional counters in their focal planes. The LECS uses an identical concentrator system but utilizes an ultra-thin entrance window to extend the response down to $0.1 \mathrm{keV}$. The non-imaging PDS consists of four independent units arranged in pairs each having a separate collimator. Each collimator was alternatively rocked on-source and $210^{\prime}$ off-source every $96 \mathrm{~s}$ during the observation. The events files were produced using the SAXDAS 2.0.0 data analysis package. LECS and MECS spectra were extracted from circular regions centered on the on-axis position of the source using radii of $8^{\prime}$ and $4^{\prime}$ respectively. The exposure times in the LECS, MECS, and PDS instruments are $14.7 \mathrm{ksec}, 34.0 \mathrm{ksec}$, and $16.4 \mathrm{ksec}$, respectively. Background subtraction for the imaging instruments was performed using standard files, but is not critical for such a bright source. Background subtraction for the PDS was carried out using data obtained when the collimator was offset from the source. The field of view of the PDS (1.3 FWHM) contains the cluster of galaxies A 3574, which is a weak X-ray emitter (Pierre et al. 1994) and the point source S3 (Madejski et al. 1995) which lies at about $12^{\prime}$ from the target. However, their 
contribution to the PDS signal is negligible (Perola et al. 1999).

\section{Integrated flux and temporal behaviour}

The spectral analysis of the IC 4329A XMM-Newton and BeppoSAX data (see Sect. 4) yields flux measurements in a large spectral band between $0.3 \mathrm{keV}$ and $150 \mathrm{keV}$. After correction of the galactic absorption by hydrogen column density, $N_{\mathrm{H}}$, of $4.4 \times 10^{20} \mathrm{~cm}^{-2}$, the fluxes in the low $(0.4-2 \mathrm{keV})$ and medium $(2-10 \mathrm{keV})$ energy bands are $F_{\mathrm{LE}}=4.2 \times 10^{-11} \mathrm{erg} \mathrm{cm}^{-2} \mathrm{~s}^{-1}$ and $F_{\mathrm{ME}}=$ $16 \times 10^{-11} \mathrm{erg} \mathrm{cm}^{-2} \mathrm{~s}^{-1}$, respectively. After correction for intrinsic absorption by material surrounding the central $\mathrm{X}$-ray source in IC $4329 \mathrm{~A}$, these correspond to luminosities of $L_{\mathrm{LE}}=6.1 \times 10^{43} \mathrm{erg} \mathrm{s}^{-1}$ and $L_{\mathrm{ME}}=8.3 \times 10^{43} \mathrm{ergs} \mathrm{s}^{-1}$ for $z=0.016$ and $H_{0}=75 \mathrm{~km} \mathrm{~s}^{-1} \mathrm{Mpc}^{-1}$. These values are similar to values in the literature which fall within $50 \%$ of $10^{-10} \mathrm{erg} \mathrm{cm}^{-2} \mathrm{~s}^{-1}$ corrected for absorption, corresponding to $L_{\mathrm{ME}}=5 \times 10^{43} \mathrm{ergs} \mathrm{s}^{-1}$ $\left(H_{0}=75 \mathrm{~km} \mathrm{~s}^{-1} \mathrm{Mpc}^{-1}\right)$. With a $2-10 \mathrm{keV}$ flux of $16 \times 10^{-11} \mathrm{erg} \mathrm{cm}^{-2} \mathrm{~s}^{-1}$, IC $4329 \mathrm{~A}$ is the second brightest hard X-ray Seyfert galaxy after NGC 4151.

A linear regression to the light curves indicates (see Fig. 1) a slight variation of the source flux over the $28.8 \mathrm{ksec}$ observation period. The EPIC $\mathrm{p}-\mathrm{n}$ count rates decreased by $2.6 \pm 0.9 \%$ and $2.5 \pm 0.7 \%$ over $11 \mathrm{ksec}$ in the $0.3-2 \mathrm{keV}$ and $2-10 \mathrm{keV}$ energy ranges, respectively. The corresponding variation in MOS count rates are respectively $3.7 \pm 1.0 \%$ and $4.5 \pm 1.9 \%$, confirming that the flux decrease was real. The flux variation between the low and high energy bands did not differ significantly. This behavior is similar to previous reports in the literature which show a modest variability of IC $4329 \mathrm{~A}$ on a time scale of hours-days.

\section{Spectral analysis}

\subsection{Phenomenological model of the Fe K spectral features}

The main spectral feature observed within the EPIC spectra of IC $4329 \mathrm{~A}$ is the Fe $\mathrm{K}$ fluorescence line at $6.4 \mathrm{keV}$. Comparison of the data with a power law model which best fit the spectral ranges around the line $(3-4.5 \mathrm{keV}$ and $8-10 \mathrm{keV}$ ) shows excess emission above the Fe K line up to an energy of about $7.0 \mathrm{keV}$ (see Fig. 2) in the observed reference frame. This feature, not reported before, suggests the presence of an absorption edge. Based on these initial observations, we fit the EPIC data in the high energy range above $3 \mathrm{keV}$ by a phenomenological model consisting of a power law continuum, a Gaussian emission line and an absorption edge. The best fit model to the EPIC $\mathrm{p}-\mathrm{n}$ data points to the existence of an iron $\mathrm{K} \alpha \mathrm{flu}-$ orescence line at $6.42_{-0.03}^{+0.04} \mathrm{keV}$ and an absorption edge at $7.1 \pm 0.4 \mathrm{keV}$ in the source rest frame (see Table 2). The absorption edge which is shallow $(\tau \approx 0.03)$ is only detected in the EPIC $\mathrm{p}-\mathrm{n}$ spectrum since the EPIC MOS, LECS
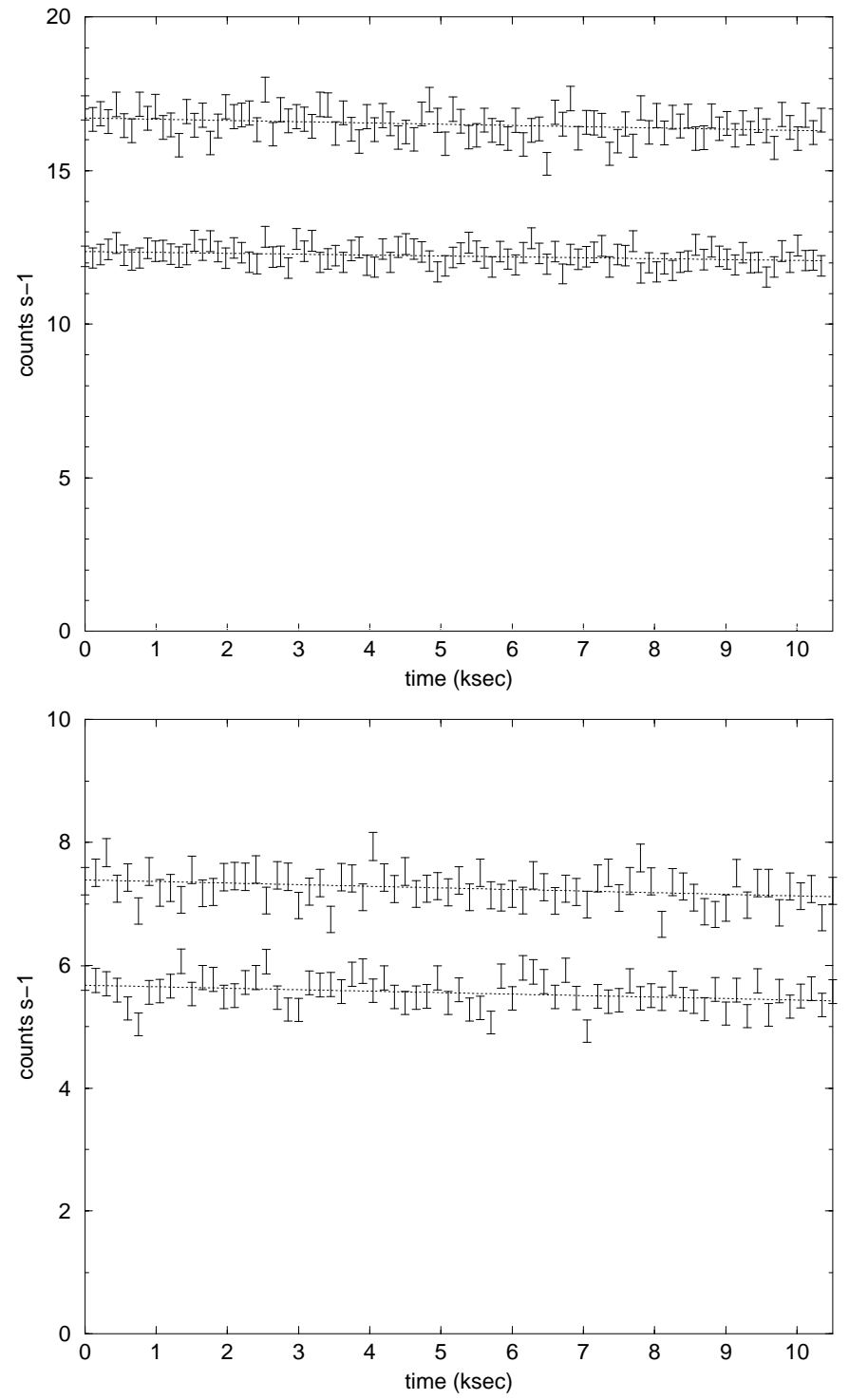

Fig. 1. Light curves of IC 4329A obtained with the EPIC p-n (top) and averaged over the EPIC MOS (bottom) cameras in the $0.3-2 \mathrm{keV}$ (upper curve) and in the $2-10 \mathrm{keV}$ (lower curve) energy bands. The noise rate within the overal band band is negligeable. The events are binned in $110 \mathrm{~s}(\mathrm{p}-\mathrm{n})$ and $150 \mathrm{~s}$ (MOS) time intervals. The slight and steady rate decreases within the observation period are illustrated by the dotted lines obtained by linear regressions applied to the light curves.

and MECS instruments have significantly lower collecting areas than the EPIC $\mathrm{p}-\mathrm{n}$ instrument.

The iron $\mathrm{K} \alpha$ fluorescence line consists of two components $\mathrm{K} \alpha_{1}$ and $\mathrm{K} \alpha_{2}$ at $6.404 \mathrm{keV}$ and $6.391 \mathrm{keV}$ respectively for Fe I with a branching ratio of 2:1 (Bambynek et al. 1972). The natural width of the lines $(\Delta E \approx 3.5 \mathrm{eV})$ and any broadening due to thermal motions of the emitting atoms $\left(\Delta E(\mathrm{eV}) \approx 0.4\left(T / 10^{6}\right)^{1 / 2}\right)$ are negligible compared to the energy resolution $(155 \mathrm{eV} \mathrm{FWHM)}$ of the EPIC camera. The mean Fe K $\alpha$ fluorescent line energy is an increasing function of ionization state. It rises slowly from $6.40 \mathrm{keV}$ with Fe I to $6.45 \mathrm{keV}$ in Fe XVII (neon-like) and then increases steeply with the escalating 
Table 1. IC 4329A fluxes measured in the $0.4-1 \mathrm{keV}$ band (RGS), 1-2 keV (RGS+LECS), 2-10 keV (MECS) and 10-150 keV (PDS) using the best fit models decribed in Sect. 4. EPIC data were not used for absolute flux measurements due to pile-up effects in the cores of the telescope point spread functions.

\begin{tabular}{ccccc}
\hline Flux $\left(\mathrm{erg} \mathrm{s}^{-1} \mathrm{~cm}^{-2}\right)$ & $0.4-1 \mathrm{keV}$ & $1-2 \mathrm{keV}$ & $2-10 \mathrm{keV}$ & $10-150 \mathrm{keV}$ \\
\hline Measured & $7.90 \pm 0.08 \times 10^{-12}$ & $3.44_{-0.21}^{+0.14} \times 10^{-11}$ & $1.64 \times 10^{-10}$ & $3.14 \times 10^{-10}$ \\
w/o Galactic absorption & $9.66 \pm 0.10 \times 10^{-12}$ & $3.80_{-0.21}^{+0.15} \times 10^{-11}$ & $1.64 \times 10^{-10}$ & $3.14 \times 10^{-10}$ \\
w/o intrinsic absorption & $68.2 \pm 0.7 \times 10^{-12}$ & $5.55_{-0.04}^{+0.07} \times 10^{-11}$ & $1.70 \times 10^{-10}$ & $3.14 \times 10^{-10}$ \\
\hline
\end{tabular}

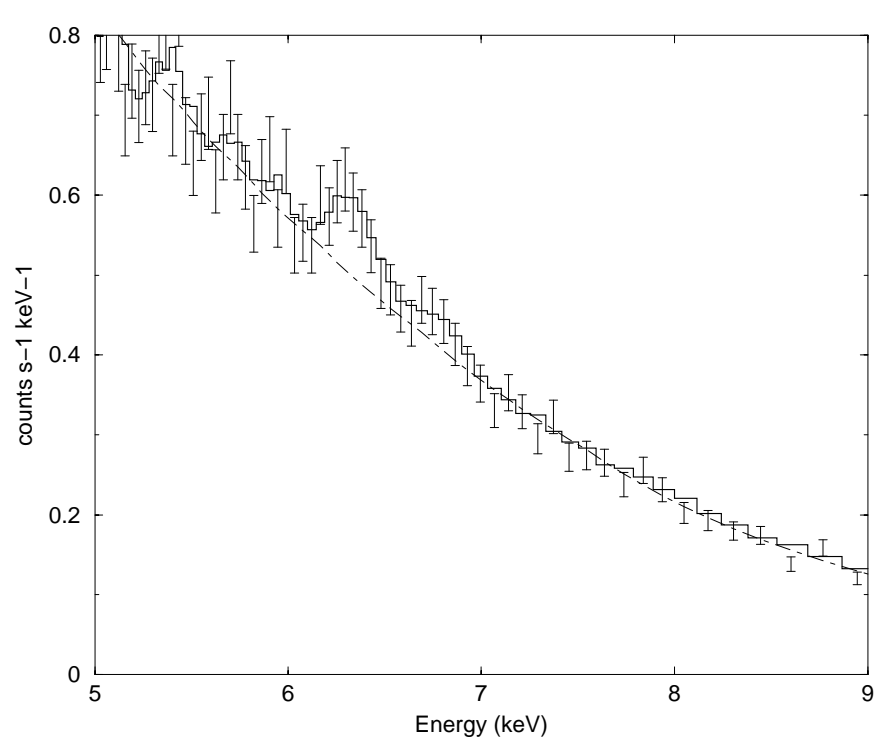

Fig. 2. Comparison of EPIC $\mathrm{p}-\mathrm{n}$ data with the power law model (dot-dashed line) which best fits the 3 to $4.5 \mathrm{keV}$ and the 8 to $10 \mathrm{keV}$ energy range around the Fe K complex. The solid line represents a running average over 4 bins with a minimum of 240 counts per bin. A significant spectral feature is evident between $6.3 \mathrm{keV}$ up to $7 \mathrm{keV}$ in the observer rest frame.

Table 2. Gaussian line and absorption edge parameters of a phenomenological model of IC 4329A EPIC spectra in the 3-10 keV spectral band.

\begin{tabular}{ccc}
\hline Model Parameters & EPIC MOS1+2 & EPIC p-n \\
\hline Edge energy $(\mathrm{keV})$ & No detection & $7.1 \pm 0.4$ \\
Edge abs. depth & No detection & $0.03 \pm 0.02$ \\
Line energy $(\mathrm{keV})$ & $6.41 \pm 0.02$ & $6.43 \pm 0.03$ \\
Line $\sigma(\mathrm{keV})$ & $0.02 \pm 0.06$ & $0.01 \pm 0.05$ \\
$\chi^{2}$ & $518 / 469$ & $91 / 109$ \\
\hline
\end{tabular}

number of vacancies in the L-shell to $6.67 \mathrm{keV}$ in Fe XXV and $6.9 \mathrm{keV}$ in Fe XXVI (House 1969; Makishima 1986). Hence, the measured energy position of the IC 4329A Fe K line at $6.42_{-0.03}^{+0.04} \mathrm{keV}$ indicates that iron is in low states of ionization, say $<$ Fe XVII.

The energy of the Fe absorption edge is also function of the ionization stage of the Fe element. Aside from any blurring effect by the detector spectral response, the iron $\mathrm{K}$ edge is not expected to be sharp due to electron scattering and due to the presence of the iron $K \beta$ line at $7.06 \mathrm{keV}$ for Fe I. Hence, the exact measurement of its energy is difficult. In the case of Fe I, the K-shell absorption edge is at an energy $E_{\mathrm{K}}=7.1 \mathrm{keV}$, rising to $7.8 \mathrm{keV}$ for Fe XVIII, and $9.3 \mathrm{keV}$ for Fe XXVI (Morita $\&$ Fujita 1983). The measured energy of the IC 4329A Fe $\mathrm{K}$ absorption edge around $7.1 \mathrm{keV}$ in the $\mathrm{p}-\mathrm{n}$ data is consistent with the presence of iron in low states of ionization $<\mathrm{Fe}$ XVII. The energy positions of the Fe K spectral features suggest that reflection from cold optically thick material is a candidate model to explain the $X M M-N e w t o n$ and BeppoSAX data above $1 \mathrm{keV}$. In the case of IC 4329A, Cold means that iron could be essentially neutral with little contribution from ionized material. It is however not excluded that $\mathrm{H}$ and $\mathrm{He}$ could be fully ionized. X-ray reflection upon an optically thick slab of cool material could also explain the high energy tail that is detected by the BeppoSAX PDS instrument in the 15-130 keV energy range (see Fig. 3).

\subsection{Reflection model of the continuum above $1 \mathrm{keV}$}

Based on the result of the phenomenological analysis, we fitted the IC 4329A spectra with a PEXRAV model (Magdziarz \& Zdziarski 1995) combined with a redshifted Gaussian component to represent the Fe K emission line. PEXRAV models an exponentially cut-off power law spectrum reflected from neutral material in a plane parallel slab. The output spectrum is the sum of the cut-off power law described by its photon index and folding energy and of the reflection component. This component is parametrized by the inclination angle to the line of sight of the reflecting slab and by the reflection fraction, $\Omega / 2 \pi$, where $\Omega$ is the solid angle subtended by the accretion disk from the X-ray source. In addition to the Galactic absorption, photoelectric absorption by a redshifted neutral column of gas was modeled using the WABS model (Morrison \& McCammon 1983). Zdziarski et al. (1994) investigated whether the spectrum of IC 4329A could be explained by partial covering with cold material rather than by reflection. They ruled out that option based on the small equivalent width of the $\mathrm{Fe} \mathrm{K}$ line generated by such a model compared with the $110 \mathrm{eV}$ measurement reported by Madejski et al. (1995). We also found that a model with partial covering by cold material gives a poorer spectral fit to IC 4329A data.

The combined XMM-Newton EPIC spectra and the BeppoSAX LECS, MECS and PDS spectra were fitted together above $1 \mathrm{keV}$ with the absorbed PEXRAV model. The $N_{\mathrm{H}}$ of the redshifted absorption, the photon-index, 
Table 3. Absorbed PEXRAV + ZGAUSSIAN models of the IC 4329A spectrum in the 1-150 keV energy range. The best fit parameters have been derived from a simultaneous fit to the XMM-Newton EPIC $\mathrm{p}-\mathrm{n}$ and MOS spectra and the BeppoSAX LECS, MECS and PDS spectra. The columns of the table describes best fit models obtained for disk inclination angles of $30^{\circ}$ (Model A) and $60^{\circ}$ (Model B).

\begin{tabular}{ccc}
\hline Parameters & Model A & Model B \\
\hline Galactic absorption & $4.4 \times 10^{20} \mathrm{~cm}^{-2}$ & $4.4 \times 10^{20} \mathrm{~cm}^{-2}$ \\
Redshifted absorption & $(42 \pm 2) \times 10^{20} \mathrm{~cm}^{-2}$ & $(42 \pm 2) \times 10^{20} \mathrm{~cm}^{-2}$ \\
Redshift & 0.016 & 0.016 \\
Disk Inclination & $30^{\circ}$ & $60^{\circ}$ \\
Photon index & $1.93 \pm 0.03$ & $1.93 \pm 0.03$ \\
Cutoff energy & $270 \pm 120 \mathrm{keV}$ & $240 \pm 100 \mathrm{keV}$ \\
Reflected fraction & $1.1 \pm 0.3$ & $1.6 \pm 0.4$ \\
Redshift & 0.016 & 0.016 \\
Fe abundance & $1.2 \pm 0.4$ & $1.3 \pm 0.5$ \\
$\chi^{2}$ & $1688 / 2564$ d.o.f. $=0.66$ & $1688 / 2564$ d.o.f. $=0.66$ \\
\hline
\end{tabular}

the folding energy, the abundances, the reflection fraction and the normalization factors were left as free parameters. A PDS normalization factor relative to the MECS of 0.7 was obtained. The redshift was fixed to 0.016 and the Galactic hydrogen column density was frozen at $4.4 \times 10^{20} \mathrm{~cm}^{-2}$. The narrow Fe $\mathrm{K}$ line near $6.4 \mathrm{keV}$ was represented by a redshifted Gaussian line. The EPIC data were fitted in the 1 to $10 \mathrm{keV}$ range and the BeppoSAX data were used in the $1-4 \mathrm{keV}$ (LECS), $1.85-10.5 \mathrm{keV}$ (MECS) and 15-150 keV (PDS) energy ranges. Fitting tests were performed using two different PEXRAV inclination angles of $30^{\circ}$ and $60^{\circ}$, respectively. A $60^{\circ}$ disk inclination angle, which is relatively large for a Seyfert 1 galaxy, was tested based on the fact that the host galaxy is seen almost edge on (Petre et al. 1984). This hypothesis is also suggested by the high $N_{\mathrm{H}}$ to IC $4329 \mathrm{~A}$ of $42 \pm 2 \times 10^{20} \mathrm{~cm}^{-2}$, i.e. strongly in excess of the Galactic value (Dickey \& Lockman 1997). Table 3 shows that the best-fit parameters obtained for disk inclination angles of $30^{\circ}$ and $60^{\circ}$ are not significantly different. The PEXRAV cut-off power law is characterized by a photon index of $1.93 \pm 0.03$ and a folding energy of $260 \pm 130 \mathrm{keV}$.

The Fe abundance in the reflecting material is poorly constrained and the amount of reflection is known to be strongly coupled to the elemental abundances (see e.g., George \& Fabian 1991; Matt et al. 1991). This is illustrated in Fig. 4 which shows confidence contours for the Fe abundance versus reflection fraction, calculated for an inclination angle of $30^{\circ}$. Although the Fe abundance and reflection fraction are not well constrained, it is worth noting that the PEXRAV model is consistent with a reflection fraction close to unity and an Fe abundance not significantly different from the cosmic value. A reflection fraction of 1.0 corresponds to an X-ray source above a centrally illuminated disk subtending a solid angle of $2 \pi \mathrm{sr}$.

The profiles of lines emitted from an accretion disk can be strongly modified by relativistic effects (Fabian et al. 1989; Stella 1990). Replacing the Gaussian model of the Fe K line by a DISKLINE (Fabian et al. 1989) or a LAOR (Laor 1991) phenomenological model does not improve the fit quality. Since, in the above tests, the relativistic smearing is only applied to the iron line and not to the reflection continuum, we also used the REFSCH+DISKLINE model. In this model, an exponentially cut-off power-law spectrum reflected from an ionized relativistic accretion disk (Magdziarz \& Zdziarski 1995) is convolved with a relativistic disk line profile. The presence of relativistic effects described by the REFSCH+DISKLINE model could not be constrained by our data. The Fe $\mathrm{K}$ line profile of IC $4329 \mathrm{~A}$ is not significantly broader (see Table 2) than the EPIC energy resolution at $6.4 \mathrm{keV}$. According to the computation of Fabian et al. (1989) and Matt et al. (1992a,b), the Fe K $\alpha$ line produced by an accretion disk is expected to be narrowest when the disk is seen face on. At large inclination angles, the contribution of gravitational and second order Doppler shifts to the line width can be substantial, depending of the inner radius of the disk. Since the Fe K line observed in the IC 4329A spectrum is narrow, it is unlikely that it includes any significant contribution from material with relativistic velocities in a strong gravitational field.

The equivalent width, $W_{\alpha}$, of the $\mathrm{K} \alpha$ line depends on several factors (Matt et al. 1991), including the iron abundance, the inclination and size of the disk and the geometry of the primary photon source. Hence, a determination of the iron abundance from $W_{\alpha}$ is subject to the uncertainties on the other parameters, not to mention the complications associated with the effects of time delays in the response of the reprocessed radiation to variations in the primary source. In the case of a semiisotropically illuminated slab of cold matter with a cosmic abundance, George \& Fabian (1991) calculated that $W_{\alpha}$ varies approximately linearly from 150 to $90 \mathrm{eV}$ for powerlaw slopes ranging from 1.2 to 2.1 , respectively. The measured $43 \pm 1 \mathrm{eV}$ (EPIC $\mathrm{p}-\mathrm{n}$ ) equivalent widths of the Fe K line and the $1.93 \pm 0.03$ slope of the IC $4329 \mathrm{~A}$ primary continuum are inconsistent with such a model. We verified that the fit compliance to the data degrades for higher equivalent width values when the reflection fraction is fixed to 1.0 and the disk inclination to a small 


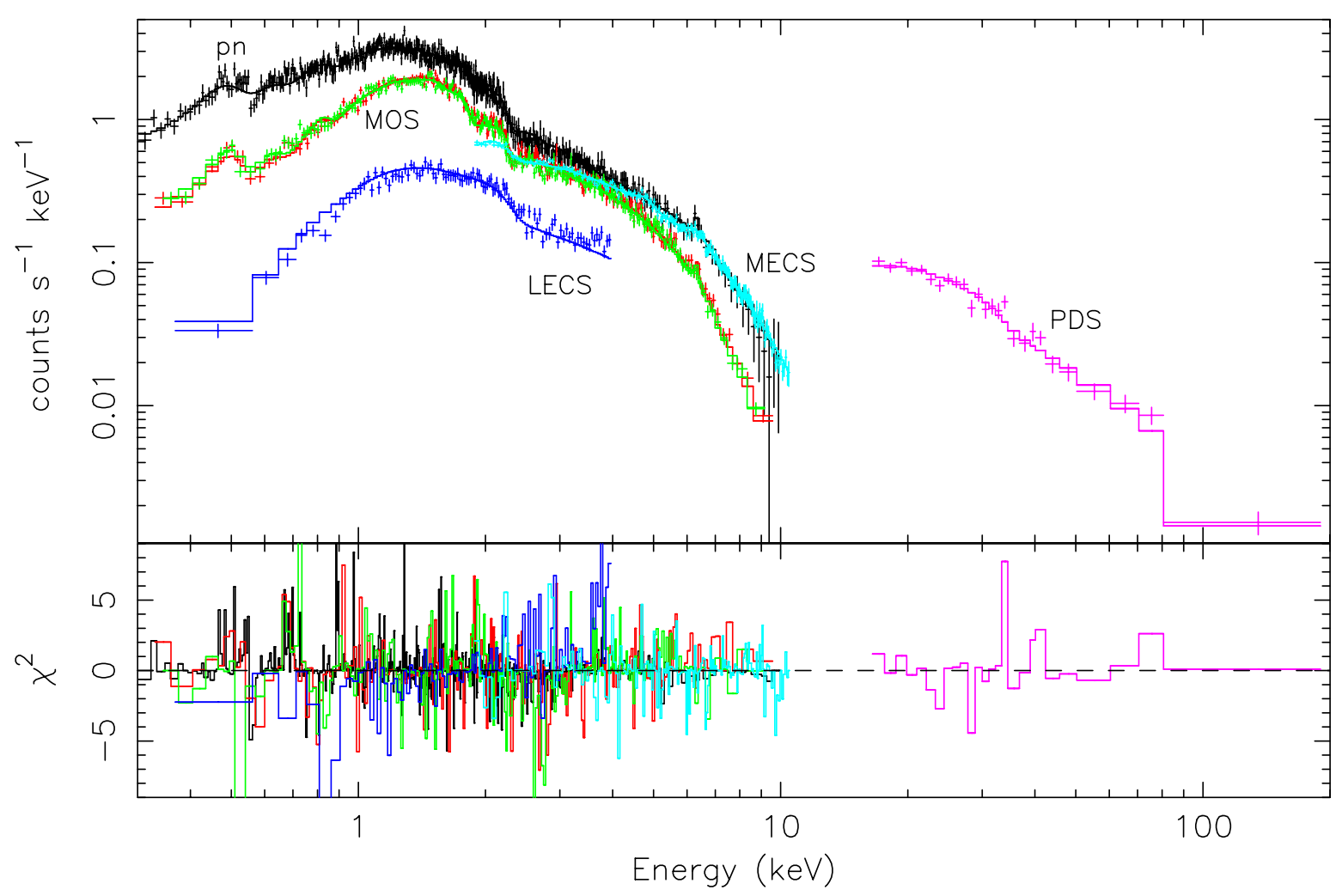

Fig. 3. Simultaneous fit of EPIC pn, EPIC MOS1, EPIC MOS 2, LECS, MECS and PDS spectra by an absorbed reflection model (see Table 3, Sect. 4.2) with two absorption edges (see Table 4, Sect. 4.3). The data and spectral fit are shown in the upper panel. The $\chi^{2}$ distributions are displayed in the lower panel.

value $(\cos i=0.95)$. However, the $W_{\alpha}$ values derived by George \& Fabian (1991) were calculated by integrating the escaping fluorescent photons over $2 \pi$ sr. They are therefore not what an observer would detect under a large disk aspect angle. Also, the atomic cross sections of Fe are uncertain. Zycki \& Czerny (1994) gives an equivalent width with respect to the total continuum of about $75 \mathrm{eV}$ for a reflection fraction of 1 , a face-on inclination, and a photon index of 1.9. Our derived value $W_{\alpha}=43 \mathrm{eV}$ would then correspond to a reflection fraction of 0.6. Since the BeppoSAX values of $\Omega / 2 \pi(=R)$ compiled by Matt (2001) are in general larger than those derived from other missions, e.g. XTE or Ginga (Lubinski \& Zdziarski 2001), our measured value $R=1.1$ might not be severely inconsistent with the strength of the Fe K $\alpha$ line predicted by such disk models for a face-on inclination. In the case of a centrally illuminated disc suggested by the best-fit PEXRAV model, the height of the source with respect to the inner disc radius contributes to reducing the equivalent width of the $\mathrm{Fe} \mathrm{K} \alpha$ line, thus preventing any conclusion regarding iron abundance from being made (George \& Fabian 1991).

\subsection{The soft (0.4-2.1 keV) energy range}

Below $1 \mathrm{keV}$, simultaneous fitting of the EPIC MOS, EPIC $\mathrm{p}-\mathrm{n}$, LECS and MECS data does not lead to acceptable $\chi^{2}$ due to the presence of instrument edges. The spectral analysis of bright sources requests that such edges are

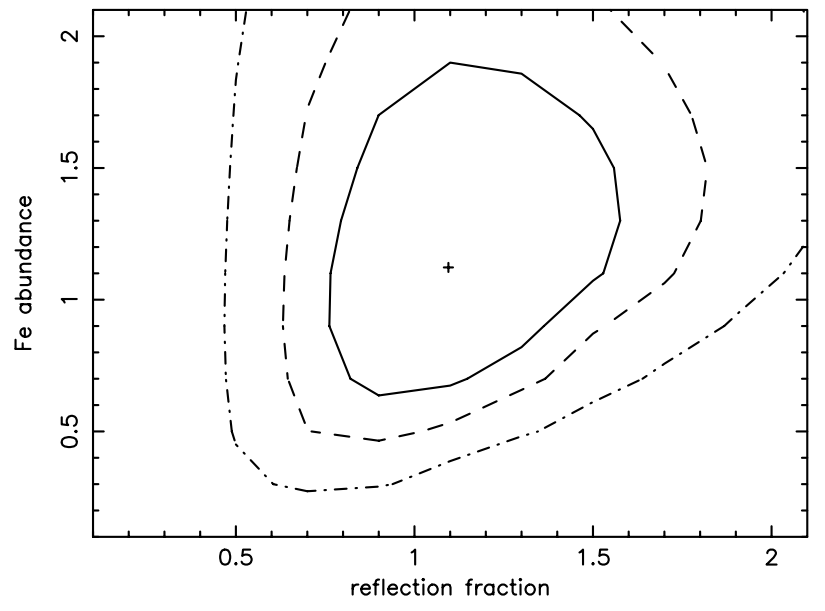

Fig. 4. Contours corresponding to $68 \%$ (solid line), $90 \%$ (dashed line) and 95\% (dot-dashed line) confidence levels for Fe abundance in the PEXRAV model versus reflection fraction. The curves have been obtained using EPIC MOS and $\mathrm{p}-\mathrm{n}$ and BeppoSAX LECS, MECS and PDS data for an assumed disk inclination of $30^{\circ}$.

calibrated with a very high accuracy, which is specially difficult on non-dispersive instruments. The reflection grating spectrometers RGS on board XMM-Newton are less sensitive to detectors, windows and filters calibration residuals for edge identification since energies are determined from the spatial position of the diffracted photons onto the CCDs. Also, the RGS instruments allow the study 


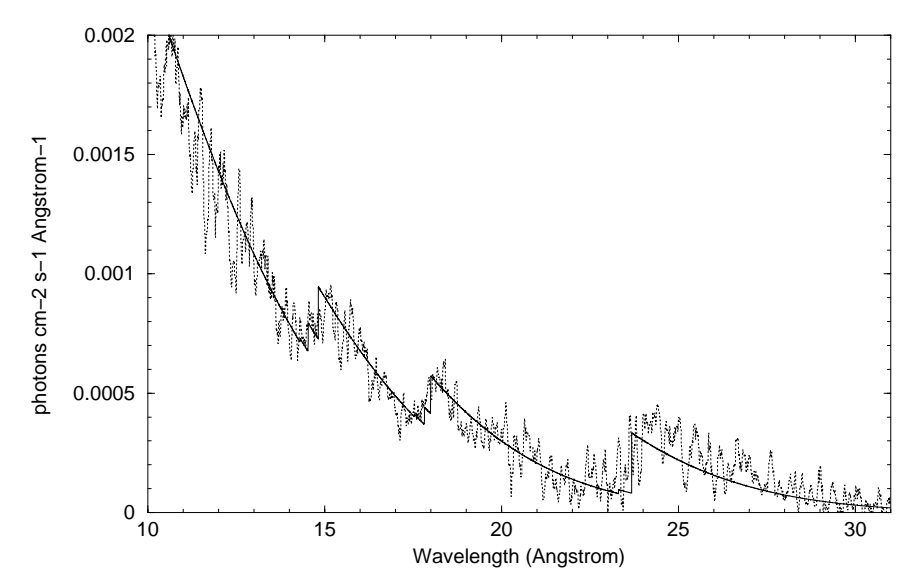

Fig. 5. First order averaged RGS spectrum compared with a best fit phenomenological model consisting of a redshifted power law absorbed by Galactic and redshifted neutral gas with solar abundance and two redshifted edges (see Table 4).

of bright sources such as IC 4329A with a higher spectral resolution. Figure 5 shows the low energy spectrum of IC 4329A obtained by averaging the 2 RGS first order spectra. The main spectral characteristics are absorption features at $15 \AA, 18 \AA$ and $24 \AA$, reminiscent of absorption edges produced by neutral and ionized oxygen.

Based on this observation, we fitted the summed RGS spectrum of IC $4329 \mathrm{~A}$ with a phenomenological model consisting of (i) a redshifted power law representing the primary continuum, (ii) two redshifted absorption edges with opacity proportional to $E^{-3}$, (iii) an intrinsic absorption by neutral matter with cosmic abundance in the rest frame of the source and (iv) a Galactic absorption fixed at the level determined by the H I $21 \mathrm{~cm}$ measurements of $4.4 \times 10^{20} \mathrm{~cm}^{-2}$. The photon index of the power law continuum was frozen to the value derived from the best fit reflection models to the LECS, MECS, EPIC and PDS spectra above $1 \mathrm{keV}$ (see Table 3). The energies and optical depths of the edges were left as free parameters. The best fit values in the source reference frame are given in Table 4 . The neutral oxygen $\mathrm{O} \mathrm{I}$ at $0.54 \mathrm{keV}$ is fitted using the WABS model. The intrinsic absorption by neutral matter is well constrained by the optical depth of the O I edge. It leads to $N_{\mathrm{H}}=(35.6) \pm 0.6 \times 10^{20} \mathrm{~cm}^{-2}$ along the line of sight to the IC $4329 \mathrm{~A}$ nucleus. Due to the use of additional edge models, this value is slightly lower than the intrinsic continuum absorption measured by the EPIC, LECS and MECS instruments above $1 \mathrm{keV}$ (see Table 3) using the WABS model alone.

The energy of the edge at $0.854 \pm 0.009 \mathrm{keV}$ is consistent with absorption by hydrogen-like oxygen O VIII at $0.871 \mathrm{keV}$. The energy of the third edge at $0.660 \pm$ $0.008 \mathrm{keV}(1 \sigma)$ is most likely related to absorption by O VI $(0.671 \mathrm{keV})$ and N VII $(0.667 \mathrm{keV})$, although a contribution from O VII $(0.739 \mathrm{keV})$ is not excluded. Since an O VIII fluorescent line can be expected at $0.65 \mathrm{keV}$, we verified that the $0.66 \mathrm{keV}$ spectral feature cannot be fitted by a Gaussian emission profile. The presence of K shell absorption edges of ionized material in the soft X-ray spectra
Table 4. Best fit phenomenological model to RGS data consisting of a redshifted power law absorbed by galactic and redshifted neutral gas with solar abundance and two redshifted edges.

\begin{tabular}{cc}
\hline Parameter & Best fit value \\
\hline Galactic absorption & $4.4 \times 10^{20} \mathrm{~cm}^{-2}$ \\
Redshifted absorption & $(35.6 \pm 0.6) \times 10^{20} \mathrm{~cm}^{-2}$ \\
Power law photon index & 1.93 \\
Redshift & 0.016 \\
Edge energy $(\mathrm{keV})$ & $0.660 \pm 0.008$ \\
Edge abs. depth & $0.43 \pm 0.04$ \\
Edge energy $(\mathrm{keV})$ & $0.854 \pm 0.009$ \\
Edge abs. depth & $0.34 \pm 0.03$ \\
$\chi^{2}$ & $4688 / 5474$ d.o.f. $=0.86$ \\
\hline
\end{tabular}

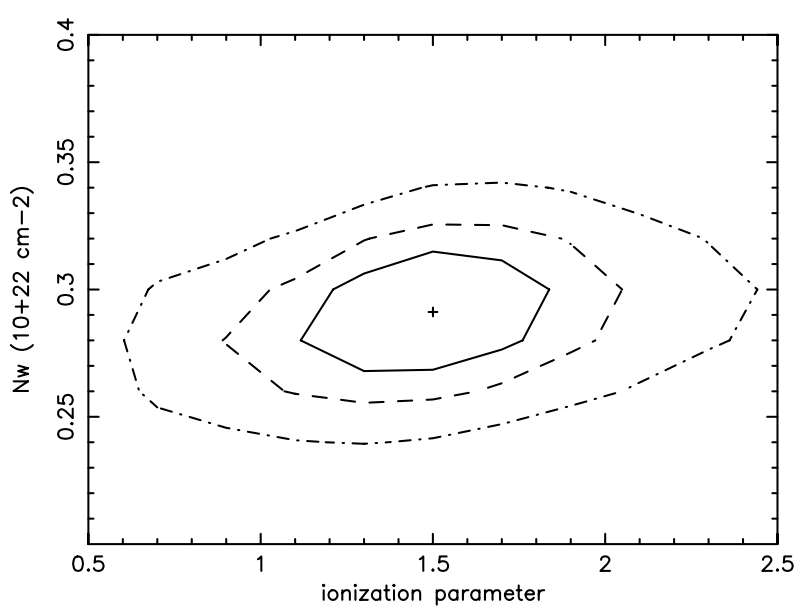

Fig. 6. RGS contours corresponding to $68 \%$ (solid line), $90 \%$ (dashed line) and 95\% (dot-dashed line) confidence levels for the $N_{W}$ of the ionized plasma and the ionization parameter $(\zeta)$.

of IC 4329A suggests the existence of optically thin, ionized material along the line of sight to the nucleus, the so-called warm absorber. We note that the characteristic energy obtained for the O VIII edge is redshifted by $17 \pm 9 \mathrm{eV}$ with respect to its rest positions in the source reference frame.

In order to quantify, in the frame of a single zone equilibrium ionization model, the column density of the ionized plasma, $N_{\mathrm{W}}$, and the ionization parameter, $\zeta$, of the warm absorber, we fitted the RGS spectrum with a model where the two edges of the phenomenological description are replaced by the XSPEC model ABSORI. In this model, the opacity of the gas is based on the ionization distribution of the relevant atomic species in a slab of Thomson-thin plasma as a function of the ionization parameter which is defined as $\zeta=L / n R^{2}$, where $n$ is the number density of the warm plasma and $R$ the distance from the ionizing source with isotropic luminosity $L$ in the interval $5 \mathrm{eV}$ to $20 \mathrm{keV}$. The photon index of the incident power law continuum was frozen to the value derived from the best fit reflection models to the LECS, MECS, EPIC 
Table 5. IC $4329 \mathrm{~A}$ spectrum in the $0.4-2.1 \mathrm{keV}$ compared with a ionized absorber model developed by Magdziarz \& Zdziarski (1995) with photoionization rates from Reilman \& Manson (1979). The photon index was frozen to the value obtained above $1 \mathrm{keV}$.

\begin{tabular}{cc}
\hline \hline Parameter & Best fit value \\
\hline Galactic absorption & $4.4 \times 10^{20} \mathrm{~cm}^{-2}$ \\
Redshifted absorption & $(30.8 \pm 0.7) \times 10^{20} \mathrm{~cm}^{-2}$ \\
Redshift & 0.016 \\
Photon index & 1.93 \\
$N_{\mathrm{W}}$ & $29 \pm 2 \times 10^{20} \mathrm{~cm}^{-2}$ \\
Temperature & $2.4 \pm 1.0 \times 10^{5} \mathrm{~K}$ \\
$\zeta$ & $1.4 \pm 0.4 \mathrm{erg} \mathrm{s}^{-1} \mathrm{~cm}$ \\
$\chi^{2}$ & $4667 / 5475$ d.o.f. $=0.85$ \\
\hline
\end{tabular}

and PDS spectra above $1 \mathrm{keV}$ (see Table 3). The results of the fit are given in Table 5. The RGS spectra of IC 4329A can be fitted by this one zone ionized absorber model with $\zeta \approx 1.4, T=2.5 \times 10^{5} \mathrm{~K}$ and an hydrogen column density $N_{\mathrm{W}}=(29 \pm 2) \times 10^{20} \mathrm{~cm}^{-2}$ of the ionized plasma with a $\chi^{2}$ of 0.85 . Remarkably, this value is similar to the $N_{\mathrm{H}}$ of the neutral gas. A contour plot of the $N_{\mathrm{W}}$ versus the ionization parameter indicates that the absorption edges provide tight constraints on a single zone warm absorber model (see Fig. 6).

\section{Discussion}

\subsection{The reflection components}

The large spectral coverage of the BeppoSAX instruments shows that the X-ray continuum emission of IC 4329A at high energies consists of a power law with a high energy tail. The high signal to noise ratio of the MOS and $\mathrm{p}-\mathrm{n}$ spectra on board $X M M-N e w t o n$ reveals an emission line at $6.42_{-0.03}^{+0.04} \mathrm{keV}$ and an absorption edge at $7.1 \pm 0.4 \mathrm{keV}$ that has not been reported before. Above $1 \mathrm{keV}$, an excellent fit to these spectral features is provided by reflection models of a cut-off power law with a photon index of $1.93 \pm 0.03$ and a cut off energy of $260 \pm 130 \mathrm{keV}$. These values are similar to the $1.86 \pm 0.03$ photon index and $270_{-80}^{+167} \mathrm{keV}$ cut-off energy derived from BeppoSAX observations performed in 1998 January (Perola et al. 1999). Nevertheless, the $25 \%$ increase of flux in the $2-10 \mathrm{keV}$ energy band with respect to the 1998 measurements indicates a significant variability of the IC 4329A primary continuum on a time scale of years. The comparison also suggests that the power law gets intrinsically steeper as the source brightens. The best fit models to the XMM-Newton and BeppoSAX spectra suggest that the features imprinted onto the primary power law continuum are likely the result of partial reprocessing of a primary X-ray continuum by cold, optically thick material, probably in the form of an accretion disk.

The underlying physical process responsible for the primary emission in radio-quiet AGNs is currently unclear. One likely explanation is that the emission is the result

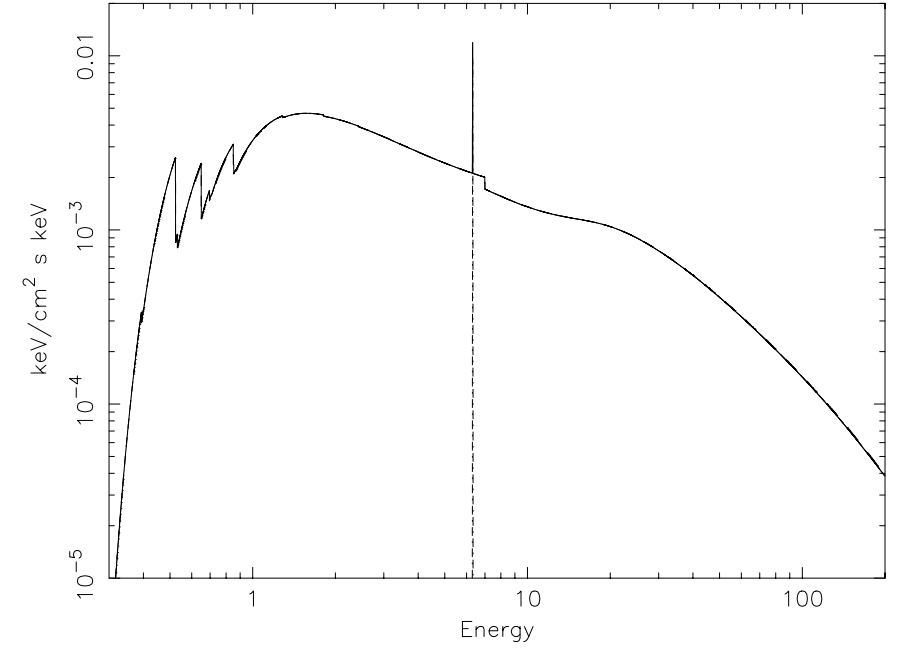

Fig. 7. $F_{\mathrm{E}}$ plot of the spectral model for IC 4329A. The model is the same as in the count rate spectrum of Fig. 3.

of inverse Compton scattering of low-energy photons by relativistic electrons. The Comptonization process could take place either on thermal electrons in an optically thin corona, on thermal electrons in the innermost part of an accretion disc or on non-thermal electrons at the base of a jet like flow. Although the geometry of the region emitting the primary X-ray spectrum is unknown, the general consensus is that the power law component originates from an extended corona in the vicinity of an accretion disc (Haardt \& Matt 1993).

Within the framework of such a model, the Fe K $\alpha$ emission and the absorption edge in the IC 4329A spectrum would be produced by Compton reflection of the primary continuum onto an accretion disk. Possible contributions to these reflection components may come from material within the accretion disk itself (Pounds et al. 1990) or from matter further out (Krolik et al. 1994; Ghisellini et al. 1994). Many Fe $\mathrm{K}$ lines are observed to be several tens of thousands of $\mathrm{km} \mathrm{s}^{-1}$ broad and significantly redshifted (Mushotzky et al. 1995; Tanaka et al. 1995; Page et al. 2001). Orbital motion deep in a relativistic gravitational potential seems to be the probable interpretation for such line profiles. The IC 4329A Fe K emission line that we measure is narrow, suggesting an origin in material located relatively far from a putative central black hole. There are, however, different ways to explain the lack of a relativistic Fe $\mathrm{K}$ line. One solution is that the inner accretion disk does not exist, but is replaced by an X-ray hot flow. Stable solutions of such an accretion flow model have been found by Narayan \& Yi (1995) which include advective as well as radiative cooling. An alternative solution is that the inner disc is present but cannot be seen in the reflected spectrum e.g., because it is fully ionized. Our result contrasts with the broadened Fe K fluorescent line observed on IC 4329A by Done et al. (2000) in 1997 August. At this time, simultaneous ASCA and RXTE spectra of IC 4329A in the $3-100 \mathrm{keV}$ range indicated that the reflection fraction for a $30^{\circ}$ disk 
inclination was lower. Although equivalenth width measurements using relativistic disk line models give higher value than Gaussian fit (Nandra et al. 1997), this suggests that the equivalent width decrease of the Fe K line since 1997 could be related to an increase of the reflection fraction. Such a behaviour is reminiscent of stratified accretion disk models (Krolik et al. 1981; Nayakshin et al. 2000) where the top Compton heated layers contribute to the reflection spectrum while the iron $\mathrm{K}$ line and edge originate from the deepest coldest regions within the disk. An increase of the reflection fraction would decrease the $\mathrm{X}$-ray flux which penetrate to the deeper cooler layers, thereby reducing directly the strength of the Fe line and edge.

The energy position of the Fe $\mathrm{K}$ line at $6.41_{-0.03}^{+0.04} \mathrm{keV}$ indicates that iron is in a low state of ionization. The gas of the reflection slab apparently remains cool despite being exposed to an intense flux of X-rays. Such a result can been interpreted as an indication that the gas in the accretion disk is dense (Guilbert \& Rees 1988; Ferland \& Rees 1988). The absorption edge at $7.1 \mathrm{keV}$ has an energy position also indicating absorption by iron atoms in low states of ionization. The strength of the Fe K absorption edge cannot be used to measure the iron abundance without an estimate of the reflected fraction of the primary continuum flux. The BeppoSAX PDS measurements of the high energy tail in the $15 \mathrm{keV}$ to $150 \mathrm{keV}$ energy range constrain the primary continuum reflection fraction to values close to one. This is consistent with a power law X-ray spectrum illuminating an optically thick, neutral disk that subtends a solid angle of $2 \pi$ sr. Compared with the 1998 BeppoSAX observation (Perola et al. 1999), our 2001 data indicates a larger amount of reflection together with a steeper spectrum and a higher flux. This result is consistent with the correlation found by Zdziarski et al. (1999) on Seyfert 1 galaxies between the amount of Compton reflection and the spectral hardness. These authors interpret this correlation as a proof that the reflecting medium play a dominant role as a source of seed soft photons for thermal Comptonization in the primary X-ray source. The larger the effective solid angle subtended by the reflector, the stronger the flux of soft photons and also, the stronger the cooling of the plasma.

Spectral fitting of the PDS measurements combined with the MOS and $\mathrm{p}-\mathrm{n}$ spectra in the $1-10 \mathrm{keV}$ range indicate that the iron abundance within the cool material reflecting the primary continuum is not significantly larger than the cosmic value. Hence, there is no evidence for iron enrichment in the accretion disk due to a special stellar evolution with e.g., a high rate of supernova explosions. Further information about the abundance and kinematics of the gas responsible for the reprocessing cannot be derived from the reflection spectrum. The disc itself may be in the form of dense clouds and the source of the hard flux may be very close to the disc and even in the intercloud medium. Composite reflected spectra have been calculated for specific geometries (George \& Fabian 1991) including a semi-isotropically illuminated slab and a centrally illuminated disc. The overall shape of the reflected spectra is predicted to be similar in both cases and cannot be used to constrain the geometry of the system.

\subsection{The warm absorber}

The presence of $\mathrm{K}$ shell absorption edges of oxygen in the soft X-ray spectra of IC 4329A suggests the existence of optically thin, ionized material along the line of sight to the nucleus, the so-called warm absorber. Photoionization is believed to dominate the physics of the warm absorbing material since an anticorrelation between the O VIII absorption depth and the primary ionized flux has been observed (Otani et al. 1996). In the case of IC 4329A, the optical depth of the O VIII edge is well constrained to $0.34 \pm 0.03$. The equivalent hydrogen column density of neutral gas is $N_{\mathrm{H}}=(35.6 \pm 0.6) \times 10^{20} \mathrm{~cm}^{-2}$. Since the absorption cross section of O VIII is $0.098 \times 10^{-18} \mathrm{~cm}^{2}$ (Ferland 1991), the column density in the ion is $N_{\text {OVIII }}=$ $(3.5 \pm 0.5) \times 10^{18} \mathrm{~cm}^{-2}$. Given the total hydrogen column density and the solar abundance of oxygen relative to hydrogen $\left(=8.51 \times 10^{-4}\right.$; Anders \& Grevesse 1989), the fraction of oxygen in hydrogen like state of ionization is $f_{\text {OVIII }}=1.2 \pm 0.2$. Hence, the absorber in IC $4329 \mathrm{~A}$ is highly ionized and has more oxygen in a hydrogen-like state than in a neutral state.

This ionized gas, which density is not constrained by the photoionization fraction alone, is expected to emit continuum and line radiation. Recombination to both O VIII and O VII leads to line emission at $0.65 \mathrm{keV}$ and $0.57 \mathrm{keV}$. Since the effective fluorescent yield of this process is about 0.5 , the absence of such line in the IC 4329A spectra suggests that the warm absorber has a low covering fraction. Also, about one third of the absorption by H-like oxygen between 0.87 and $1.2 \mathrm{keV}$ could result in Lyman continuum emission. These diffuse continuum emission features might be very sharp because of the low temperature of the photoionized gas. Their intensity, which depends on the column density and the covering factor of the gas, is not expected to be large (Netzer 1993). These lines are observed against a faint absorbed continuum with a considerably large equivalent width. Hence, unless the optical depth is great enough to absorb a significant fraction of the continuum power, the luminosity of the gas intrinsic emission will be a small fraction of the direct luminosity (Krolik \& Kriss 1995). This may explain why we do not detect emission lines in the RGS spectra of IC 4329A. Although spectral fitting should in principle include such emission processes, the moderate signal to noise ratio of the IC 4329A RGS spectra is only able to constrain simple absorber models.

Assuming optically thin clouds in a plane parallel geometry with constant hydrogen density, in ionization equilibrium and surrounding a central source of radiation, Porquet et al. (1999) found that only a restricted range of the ionization paramater, $\zeta$, and hydrogen column density, $N_{\mathrm{W}}$, can reproduce the mean $\mathrm{O}$ VII and O VIII 
optical depths of the Seyfert 1 sample studied by Reynolds (1997). The best fit values of $N_{\mathrm{W}}$ and $\zeta$ parameter derived here $\left(N_{\mathrm{H}}=31 \times 10^{20} \mathrm{~cm}^{-2}, N_{\mathrm{W}}=(29 \pm 2) \times 10^{20} \mathrm{~cm}^{-2}\right.$ and $\left.\zeta=1.4 \pm 4 \mathrm{erg} \mathrm{s}^{-1} \mathrm{~cm}\right)$ are also well constrained by a single zone absorber model. These values are comparable with the values $\left(N_{\mathrm{H}}=26_{-2}^{+8} \times 10^{20} \mathrm{~cm}^{-2}, N_{\mathrm{W}}=\right.$ $31_{-1}^{+14} \times 10^{20} \mathrm{~cm}^{-2}$ and $\left.\zeta=2.4_{-2}^{+24} \mathrm{erg} \mathrm{s}^{-1} \mathrm{~cm}\right)$ derived by Perola et al. (1999) from a BeppoSAX observation of IC 4329A performed in 1998 January. The photon index of the incident power law spectrum $\left(\Gamma=1.88_{-0.02}^{+0.04}\right)$ that was derived from the BeppoSAX observation was softer than our XMM-Newton BeppoSAX measurement (1.93 \pm 0.03$)$.

Best fit parameters obtained from spectral fitting using single zone photoionization models can hardly be regarded as measurements of true physical quantities. Various works including the spectral variability study of MCG-6-30-15 (Otani et al. 1996) and the simultaneous UV/X-ray studies of NGC 3516 (Kriss et al. 1996a,b) strongly argue for a multi-zone absorber. Our medium resolution RGS observations of IC 4329A are also remarkable in that respect since we detected one edge which coincides with a blend of O VI and N VII and one edge at O VIII. Although a contribution from O VII cannot be excluded, the simultaneous presence of O VI and O VIII edges without significant O VII absorption makes a single zone model unrealistic. Also, the state of the warm absorber in our observation appears different from that found in the 1998 BeppoSAX observations where Perola et al. (1999) found an edge corresponding to a blend of O VI and O VII but no evidence of a significant absorption at the O VIII edge location. In view of the moderate spectral resolution of the LECS compared with RGS, this supports the idea that the O VI, N VII, O VII edge complex could remain constant in time scale of years, whereas the O VIII dramatically varies in optical depth over shorter periods. This behavior would also invalidate a one zone photoionization model where the O VII and O VIII recombination and photoionization time scales are expected to be comparable since the two dominant oxygen ions are assumed to proceed from the same material.

Porquet et al. (1999) suggest the use of two-zone warm absorber models. Each zone responsible of a given edge would have a negligeable contribution to the other edge which is mainly formed in the second zone. These authors calculated, using photoionized and hybrid models, that a high density $\left(n_{\mathrm{H}} \geq 10^{10} \mathrm{~cm}^{-3}\right)$ and a high ionization parameter $(\zeta \approx$ a few hundred) is likely needed to produce the mean O VIII absorption feature of Seyfert 1 galaxies. Such a high density for $\zeta=100 \mathrm{erg} \mathrm{cm} \mathrm{s}^{-1}$ lead to a maximum radius of the warm absorbing material $R<1.7 \times 10^{16} \mathrm{~cm}(0.006 \mathrm{pc})$ using the measured intrinsic luminosity $\left(L_{\mathrm{X}} \approx 3 \times 10^{44} \mathrm{erg} \mathrm{s}^{-1}\right)$ of IC $4329 \mathrm{~A}$ in the $0.4 \mathrm{keV}$ to $130 \mathrm{keV}$ energy range. This value is within the upper limit $(0.03 \mathrm{pc})$ of the broad line region radius estimated from time delay between the visible continuum and the response of the $\mathrm{H} \alpha$ and $\mathrm{H} \beta$ line of IC 4329A (Winge et al. 1996). This result is consistent with the hypothesis that the region producing the O VIII edge could be located inside the broad line region (BLR) while the warm absorber associated with the O VI edge would be located within the BLR or further outside.

In the August 1993 ASCA observation of IC 4329A, Cappi et al. (1996) found clear evidence of two edges coinciding with O VII and O VIII redshifted by about $25 \mathrm{eV}$. Remarkably, the characteristic energy that we obtain for the O VIII edge is also redshifted by $17 \pm 9 \mathrm{eV}$ with respect to its rest positions in the source reference frame. If real, this corresponds to infall Doppler velocities of between $3000 \mathrm{~km} \mathrm{~s}^{-1}$ and $9000 \mathrm{~km} \mathrm{~s}^{-1}$. This redshift contrasts with the $3 \%$ blueshift of the O VII and O VIII absorption edges of NGC 4051 (Mihara et al. 1994) which could be due to an outflow velocity of $\approx 10000 \mathrm{~km} \mathrm{~s}^{-1}$. Nevertheless, such an interpretation is subject to uncertainties in the measured optical depth and energy of the absorption edges. In particular, blended lines may significantly shift the apparent energy of absorption edges. Resonance line opacity and scattering could affect the energy dependant opacity (Krolik \& Kriss 1995) in a way that would require the introduction of various model dependant parameters, including covering factors and widths of the material velocity distributions.

As previously indicated, the state of the warm absorber in our 2001 observation and in the 1993 ASCA observation (Cappi et al. 1996) appear different from that found in the BeppoSAX observation performed on 1998 January (Perola et al. 1999). In particular the absence of a significant absorption at the O VIII edge in these previous BeppoSAX observation implies a lower ionization parameter. This appears consistent with the fact that the intensity of the ionizing continuum at that time, as inferred from the direct primary emission, was lower than in our measurements. On the contrary, this contrasts with the fact that the ionizing continuum in this January 1998 BeppoSAX observation was 30\% higher than in the 1993 ASCA observation where an O VIII edge was detected. This suggest that delay effects due to relaxation to an equilibrium state in the ionization of the absorber should be taken into account when comparing the warm absorber state with the ionizing luminosity of the central source. Regular monitoring of IC 4329A is needed to understand the physical conditions which prevail in the ionized medium close to the central engine.

Acknowledgements. We thank our colleagues from the XMMNewton and BeppoSAX Science Operation Centers for their support in implementing the observations. We are grateful to the referee, Dr. A. Zdziarski, for the improvements suggested to an earlier version of the manuscript.

\section{References}

Anders, E., \& Grevesse, N. 1989, Geochim. Cosmochim. Acta, 53, 197

Bambynek, W., Craseman, B., Fink, R. W., et al. 1972, Rev. Mod. Phys., 44, 716 
Boella, G., Chiappetti, L., Conti, G., et al. 1997, A\&AS, 122, 327

Cappi, M., Mihara, T., Matsuoka, M., et al. 1996, ApJ, 458, 149

Dickey, J. M., \& Lockman, F. J. 1990, ARA\&A, 28, 215

Done, C., Madejski, G. M., \& Zycki, P. T. 2000, ApJ, 536, 213

Fabian, A. C., Rees, M. J., Stella, L., et al. 1989, MNRAS, 238, 729

Ferland, G. J., \& Rees, M. J. 1988, ApJ, 332, 141

Ferland, G. L. 1991, Ohio State Univ., Astron. Dept. Int. Rep., 91-01

Fiore, F., Guainazzi, M., \& Grandi, P. 1999, Cookbook for BeppoSAX NFI Spectral Analysis (www.sdc.asi.it/software/cookbook)

Frontera, F., Costa, E., Dal Fiume, D., et al. 1997, A\&AS, 122, 371

George, I. M., \& Fabian, A. C. 1991, MNRAS, 249, 352

Ghisellini, G., Haardt, F., \& Matt, G. 1994, MNRAS, 267, 743

Gondoin, P., Aschenbach, B., Erd, C., et al. 2000, SPIE Proc., 4140, 1

Gondoin, P., Lumb, D., Siddiqui, H., et al. 2001, A\&A, 373, 805

Guainazzi, M., Nicastro, F., Fiore, F., et al. 1998, MNRAS, 301, L1

Guilbert, P. W., \& Rees, M. J. 1988, MNRAS, 233, 475

Haardt, F., \& Matt, G. 1993, MNRAS, 261, 346

den Herder, J. W., Brinkman, A. C., Kahn, S. M., et al. 2001, A\&A, 365, L7

House, L. L. 1969, ApJS, 18, 21

Jansen, F., Lumb, D., Altieri, B., et al. 2001, A\&A, 365, L1

Kriss, G. A., Espey, B. R., Krolik, J. H., et al. 1996a, ApJ, 467,622

Kriss, G. A, Krolik, J. H., Otani, C., et al. 1996b, ApJ, 467, 629

Krolik, J. H., Mc Kee, C. F., \& Tarter, C. B. 1981, ApJ, 249, 422

Krolik, J. H., Madau, P., \& Zycki, P. 1994, ApJ, 420, L57

Krolik, J. H., \& Kriss, G. A. 1995, ApJ, 447, 512

Laor, A., 1991, ApJ, 376, 90

Lubinski, P., \& Zdziarski, A. A. 2001, MNRAS, 323, L37

Madejski, G. M., Zdziarski, A. A., Turner, T. J., et al. 1995, ApJ, 438, 672

Magdziarz, P., \& Zdziarski, A. A. 1995, MNRAS, 273, 837

Makishima, K, 1986, in The Physics of Accretion onto Compact Objects, ed. K. O. Mason, M. G. Watson, \& N. E. White (Springer Verlag, Berlin), 250

Matt, G., Perola, G. C., Piro, L. 1991, A\&A, 247, 25

Matt, G., Perola, G. C., Piro, L., \& Stella, L. 1992a, A\&A, 257, 63

Matt, G., \& Perola, G. C. 1992b, MNRAS, 259, 433

Matt, G., 2001, Astroph. Lett. \& Comm., in press [astro-ph/0007105]
Mihara, T., Matsuoka, M., Mushotzky, R. F., et al. 1994, PASJ, 446, L137

Miyoshi, S., Yamashita, K., Okumura, Y., et al. 1988, PASJ, 40,127

Morita, S., \& Fujita, J. 1983, J. Phys. Soc. Japan, 52, 1957

Morrison, T., \& McCammon, D. 1983, ApJ, 270, 119

Mushotzky, R. F., Fabian, A. C., Iwasawa, K., et al. 1995, MNRAS, 272, L9

Nandra, K., George, I. M., Mushotzky, R. F., et al. 1997, ApJ, 488, L91

Narayan, R., \& Yi, I. 1995, ApJ, 444, 231

Nayakshin, S., Kazanas, D., Kallman, T. R. 2000, ApJ, 537, 833

Netzer, H. 1993, ApJ, 411, 594

Otani, C., Kii, T., Reynolds, C. S., et al. 1996, PASJ, 48, 211

Page, M. J., Mason, K. O., Carrera, F. J., et al. 2001, A\&A, 365, L152

Parmar, A. N., Martin, D. D. E., Bavdaz, M., et al. 1997, A\&AS, 122, 309

Perola, G. C., Matt, G., Cappi, M., et al. 1999, A\&A, 351, 937

Petre, R., Mushotzky, R. F., Krolik, J., et al. 1984, ApJ, 280, 499

Pierre, M., Bohringer, H., Ebeling, H., et al. 1994, A\&A, 290, 725

Piro, L., Yamauchi, M., \& Matsuoka, M. 1990, ApJ, 360, L35

Pounds, K. A., Nandra, K., Stewart, G. C., et al. 1990, Nature, 344,132

Porquet, D., Dumont, A. M., Collin, S., et al. 1999, A\&A, 341, 58

Reilman, R. F., \& Manson, S. T. 1979, ApJS, 40, 815

Reynolds, C. S. 1997, MNRAS, 286, 513

Stella, L. 1990, Nature, 344, 747

Tanaka, Y., Nandra, K., \& Fabian, A. C. 1995, Nature, 375 659

Turner, T. J., \& Pounds, K. A. 1989, MNRAS, 240, 833

Turner, M. J. L. T., Abbey, A., Arnaud, M., et al. 2001, A\&A, 365, L27

Walter, R., \& Fink, H. H. 1993, A\&A, 274, 105

Weaver, K. A., Nousek, J., Yaqoob, T., et al. 1996, ApJ, 458, 160

Whittle, M., 1992, ApJS, 79, 49

Wilson, A. S., \& Penston, M. V. 1979, ApJ, 232, 389

Winge, C., Peterson, B. M., Pastoriza, M. J., et al. 1996, ApJ, 469, 648

Yaqoob, T., George, I. M., Nandra, K., et al. 2001, ApJ, 546, 759

Zdziarski, A. A., Fabian, A. C., Nandra, K., et al. 1994, MNRAS, 269, L55

Zdziarski, A. A., Lubinski, P., \& Smith, D. A. 1999, MNRAS, 303, L11

Zycki, P. T., \& Czerny, B. 1994, MNRAS, 266, 653 